\title{
Viewpoint
}

\section{A cooler way to operate atomic clocks}

\author{
By Jürgen Appel \\ Niels Bohr Institute, University of Copenhagen, Denmark
}

Published June 1, 2009

Dispersive probing of an atomic transition decreases the "dead time" of optical atomic clocks, potentially enabling more stable time reference standards.

Subject Areas: Atomic and Molecular Physics, Optics

\author{
A Viewpoint on: \\ Nondestructive measurement of the transition probability in a Sr optical lattice clock \\ Jérôme Lodewyck, Philip G. Westergaard and Pierre Lemonde \\ Phys. Rev. A 79, 061401 (2009) - Published June 1, 2009
}

Optical atomic clocks based on neutral atoms [1] are poised to become the most precise measurement instruments ever constructed. Their "tick" is based on measuring the frequency of light that drives an ultranarrow linewidth optical transition in an ensemble of strontium atoms with an excited state lifetime of more than $100 \mathrm{~s}$. Writing in Physical Review A, Jérôme Lodewyck, Philip G. Westergaard, and Pierre Lemonde at the Observatoire de Paris, France, report a new method to probe the atomic state in a strontium optical lattice clock that allows them to drastically improve the time over which frequency data can be acquired, and thus boost the potential stability and accuracy of the clock [2].

The challenge in constructing an optical clock lies in controlling external influences that perturb the frequency and even more so in actually spectroscopically resolving this narrow resonance. No light source exists yet that is as narrow as the Sr clock transition. Although construction of narrower linewidth lasers by means of the atomic clock transition itself has been proposed [3], today the most stable lasers are stabilized with the use of carefully designed high-quality optical resonators. In these cavities, the light is reflected between the mirrors approximately a million times to average out fluctuations, which yields linewidths of about $0.1 \mathrm{~Hz}[4]$. The goal in atomic clock research is to provide a reference frequency to compensate long-term drifts of such a laser, so that its optical oscillations can be counted and serve as a time reference.

A typical clock cycle involves three steps: Laser cooling, an interrogation pulse, and counting atoms. First, in order to eliminate motional effects (Doppler broadening and atomic recoil), the strontium atoms are laser cooled to their longitudinal motional ground state while an offresonant standing-wave laser beam forms a 1D optical lattice that traps the atoms and holds them in place for the actual clock operation to be performed. In the second step, a fixed-duration interrogation pulse pro-

DOI: $10.1103 /$ Physics.2.45

URL: http://link.aps.org/doi/10.1103/Physics . 2.45 duced by the ultrastable laser is sent through the cold atomic ensemble. Every other clock cycle, the interrogation pulse is alternately red- or blue-detuned by approximately half a linewidth such that half of the atoms are transferred into the long-lived excited clock state. Finally, in a third step, the number of atoms remaining in the ground state is measured. After repumping the atoms from the excited state back to the ground state and thus counting the total atom number, the excitation probability is determined.

A difference in the fraction of excited atoms during successive clock cycles of alternate laser detuning signifies a drift of the ultrastable laser, which can then be compensated for so that its optical frequency forms a precise time standard. Only during the second step of the clock operation, which takes as little as $10 \%$ of the whole cycle time, is the laser's frequency compared with that of the atoms, whereas the cooling and atom counting phases can be regarded as "dead time" as no frequency information is obtained.

This low duty cycle is caused mainly by the way the atoms are counted: Using an optical transition that is much broader than the clock transition, atoms in the ground state are excited and the fluorescence light of atoms decaying back to the ground state is then detected. Although by this method many photons can be scattered in all directions from each single atom, most photons are not registered and the photon recoil heats up the atomic cloud so that atoms eventually drop out of the trapping lattice. Therefore after each probing, a new atomic cloud has to be cooled and captured, which takes up most of the cycle time.

In their article, Lodewyck et al. present an alternative way to count the atoms without heating them out of the trap and thus they can drastically shorten the lengthy cooling step in every cycle. This results in a much more frequent comparison of the ultrastable laser with the atomic frequency. The phase noise of this laser is the 
dominating noise source in clocks todays (the so-called Dick effect [5]); it decreases towards higher sideband frequencies, so the authors expect a significant stability improvement.

When atoms interact with light, two effects occur: Light gets absorbed, and it experiences a phase shift due to the refractive index of the atoms being different from unity. For light intensities well below optical saturation, both effects happen proportionally to the number of atoms in the path of the beam. Whereas the traditional fluorescence detection is based on absorption, the authors implement a detection technique that counts atoms by measuring the phase shift imprinted on light passing through the atomic cloud.

In a two-level system the absorption probability decreases with increasing detuning $\Delta$ from resonance as $1 /\left[\Delta^{2}+\Gamma^{2}\right]$ (where $\Gamma$ is the transition linewidth) and the dispersion only decreases with $\Delta /\left[\Delta^{2}+\Gamma^{2}\right]$, and so a weak off-resonant laser beam is used to perform a shot-noise-limited phase measurement while avoiding too much heating by fluorescence. However, choosing too large a detuning does not pay off, since the probe light intensity has to increase with $\Delta^{2}$ to compensate the falling signal amplitude, so that for a fixed fluorescence rate the signal-to-shot noise ratio rapidly approaches its theoretical maximum for detunings of only a few times the linewidth $\Gamma$.

To measure the phase shift independently from optical path length fluctuations, the authors probe the atoms with a single beam that essentially contains two optical frequencies that experience opposite phase shifts due to opposite detunings with respect to the atomic resonance (Fig. 1). By interfering the transmitted light fields with a strong reference laser beam (the so-called local oscillator) in a homodyne detection setup, Lodewyck et al. are able to achieve a photon shot-noise-limited phase detection.

In fact, the authors were able to use photon numbers so high that the phase resolution approaches precision regimes where the so-called projection noise of the atomic state soon becomes visible. If the clock interrogation pulse has a 50\% chance of transferring each individual atom into the excited state, starting from $N$ atoms in the ground state, there is an intrinsic statistical uncertainty and we expect to find $N / 2 \pm \sqrt{N / 2}$ excited atoms. This uncertainty forms a classical limit for detecting the fraction of excited atoms.

To further increase the effective optical density and phase sensitivity, it has been suggested [6] that the atoms be placed into a low-finesse cavity. The phase measurement method presented by the authors is ideally suited for such an application. Since only the phase difference of the two sidebands is detected, length fluctuations of a cavity that has a free spectral range, such that it is resonant to both sidebands, do not deteriorate the measurement.

Recent research [6, 7] shows that dispersive atomic population measurements similar to the ones presented

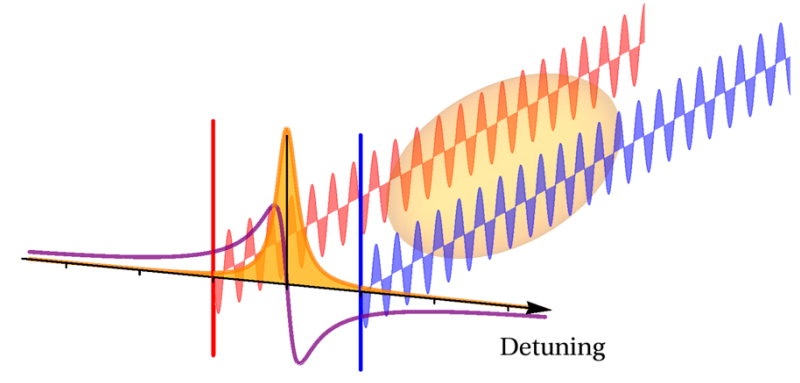

FIG. 1: A dual-color light beam passes through an atomic ensemble (orange cloud). One of the frequencies is red-detuned and the other is blue-detuned, with respect to the atomic absorption line (orange curve shows the imaginary part of the atomic susceptibility). The atoms induce opposite phase shifts on the light (purple dispersion curve shows the real part of the susceptibility), so that after passing through the ensemble the differential phase shift is proportional to the atom column density in the light path. Since both colors are significantly far from resonance, spontaneous photon scattering and troublesome heating of the atomic ensemble is strongly suppressed.

here can be used to prepare collectively entangled atomic states in optically dense ensembles, such that quantum correlations between excitations of different atoms are created that beat the classical limit. In this respect, this new detection method may open the path towards new stability regimes where the influence of noise sources like blackbody thermal radiation, or even quantum projection noise, can be better investigated.

\section{References}

[1] A. D. Ludlow, et al. Science 319, 1805 (2008).

[2] J. Lodewyck, P. G. Westergaard, and P. Lemonde, Phys. Rev. A 79, 061401 (2009).

[3] D. Meiser, J. Ye, D. R. Carlson, and M. J. Holland, Phys. Rev. Lett. 102, 163601 (2009).

[4] J. Millo et al., arXiv:0901.4717.

[5] G. J. Dick, J. D. Prestage, C. A. Greenhall, and L. Maleki, in Proceedings of the 22nd Annual Precise Time and Time Interval (PTTI) Applications and Planning Meeting, Vienna, Virginia, 1990 (NASA Conference Publication No. 3116, 1990), p. 487.

[6] M. H. Schleier-Smith, I. D. Leroux, and V. Vuletić, arXiv:0810.2582.

[7] J. Appel et al., arXiv:0810.3545. 


\section{About the Author}

Jürgen Appel

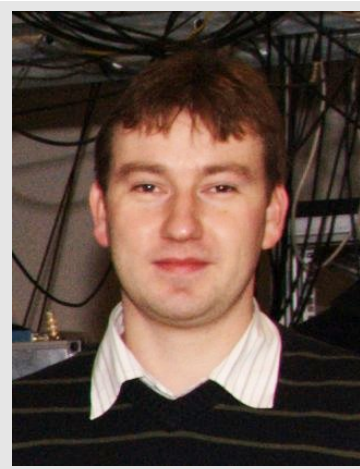

Jürgen Appel started his doctoral work on quantum optics at the University of Konstanz, Germany, in the group of Prof. A. Lvovsky, investigating quantum interaction between light and atoms by electromagnetically induced transparency (EIT). In 2004, the group moved to Calgary, Canada, and set up new laboratories at the Institute for Quantum Information Science. In June 2007, he received his Ph.D. in physics for work on producing continuous variable nonclassical light states and storing them in an EIT quantum memory. Dr. Appel is currently a postdoctoral researcher at the Niels Bohr Institute, Copenhagen, Denmark, in the group of Prof. Eugene Polzik, working on quantum nondemolition measurements, spin-squeezing on the cesium clock transition and on light-atom quantum interfaces. 